Physics

Physics Research Publications

Purdue University

Year 2006

\title{
Rigorous approach to the comparison between experiment and theory in Casimir force measurements
}

L. Klimchitskaya*

E. Fischbach**

U. Mohideen ${ }^{\S}$
F. Chen ${ }^{\dagger}$

D. E. Krause ${ }^{\dagger \dagger}$

V. M. Mostepanenko
R. S. Decca ${ }^{\ddagger}$

D. Lopez

This paper is posted at Purdue e-Pubs.

http://docs.lib.purdue.edu/physics_articles/333 


\title{
Rigorous approach to the comparison between experiment and theory in Casimir force measurements
}

\author{
G L Klimchitskaya ${ }^{1}$, F Chen ${ }^{2}$, R S Decca ${ }^{3}$, E Fischbach ${ }^{4}$, D E Krause ${ }^{5,4}$, \\ D López ${ }^{6}$, U Mohideen ${ }^{2}$ and V M Mostepanenko ${ }^{7}$
}

${ }^{1}$ North-West Technical University, Millionnaya St 5, St Petersburg, Russia

2 Department of Physics, University of California, Riverside, CA 92521, USA

${ }^{3}$ Department of Physics, Indiana University-Purdue University Indianapolis, Indianapolis, IN 46202, USA

${ }^{4}$ Department of Physics, Purdue University, West Lafayette, IN 47907, USA

5 Department of Physics, Wabash College, Crawfordsville, IN 47933, USA

${ }^{6}$ Bell Laboratories, Lucent Technologies, Murray Hill, NJ 07974, USA

${ }^{7}$ Noncommercial Partnership 'Scientific Instruments', Moscow, Russia

Received 28 October 2005, in final form 13 December 2005

Published 10 May 2006

Online at stacks.iop.org/JPhysA/39/6485

\begin{abstract}
In most experiments on the Casimir force the comparison between measurement data and theory was done using the concept of the root-mean-square deviation, a procedure that has been criticized in the literature. Here we propose a special statistical analysis which should be performed separately for the experimental data and for the results of the theoretical computations. In so doing, the random, systematic and total experimental errors are found as functions of separation, taking into account the distribution laws for each error at $95 \%$ confidence. Independently, all theoretical errors are combined to obtain the total theoretical error at the same confidence. Finally, the confidence interval for the differences between theoretical and experimental values is obtained as a function of separation. This rigorous approach is applied to two recent experiments on the Casimir effect.
\end{abstract}

PACS numbers: $12.20 . \mathrm{Fv}, 12.20 . \mathrm{Ds}, 07.05 . \mathrm{Kf}$

\section{Introduction}

Today the Casimir effect is being actively investigated not only theoretically but also experimentally. Historically the first measurement of the Casimir force between metals was performed in 1958 [1] and confirmed the existence of the force with an uncertainty of about $100 \%$. In the following decades the experimental output was painfully low and only one experiment with metal test bodies was made [2] (see [3] for a review). In the last few years many measurements of the Casimir force have been performed using torsion pendulums, atomic force 
microscopes, micromechanical torsional oscillators and other laboratory techniques [4-16]. Most authors (see [1-14]) have used the concept of the root-mean-square deviation between experiment and theory to quantify the precision of the measurements. However, for strongly nonlinear quantities, such as the Casimir force which changes rapidly with separation distance, this method is not appropriate because it may lead to different results when applied in different ranges of separations. This was emphasized in [9] although no better method was suggested.

The present paper contains the comparison analysis of the precision and accuracy in two recent experiments $[15,16]$ using rigorous methods of mathematical statistics. The distinctive feature of our approach is that both total experimental and total theoretical errors are determined independently of one another at some accepted confidence level. Then, the absolute error of differences between calculated and measured values of the physical quantity is found at the same confidence as a function of separation, serving as a measure of the precision in the comparison of experiment and theory.

\section{Determination of the experimental errors}

\subsection{Random errors}

In experiment [15], the Casimir pressure between two Au-coated parallel plates was determined dynamically by means of a microelectromechanical torsional oscillator within the separation region from 160 to $750 \mathrm{~nm}$. In the experiment [16], the Casimir force was measured between a Si plate and a large Au-coated sphere using an atomic force microscope within the separations from 62.33 to $600.04 \mathrm{~nm}$. In our error analysis, we use the notation $\Pi(z)$ which denotes either the measured Casimir pressure $P^{\exp }(z)$ or force $F^{\exp }(z)$ as a function of separation $z$ between the test bodies.

Usually several sets of measurements, say $n$, are taken within one separation region $\left(z_{a}, z_{b}\right)$. This is done in order to decrease the random error and to narrow the confidence interval. In [15] $n=14$, and in [16] $n=65$. Each set consists of pairs $\left[z_{i}, \Pi\left(z_{i}\right)\right]$, where $1 \leqslant i \leqslant i_{\max }=(288-293)$ in [15] and $1 \leqslant i \leqslant i_{\max }=3164$ in [16]. All measurement data can be represented by pairs $\left[z_{i j}, \Pi\left(z_{i j}\right)\right]$, where $1 \leqslant j \leqslant n$. Generally speaking, separations with fixed $i$ but different $j$ may be different (this was the case in [15]). For such measurement results, it is reasonable to divide the entire separation range $\left(z_{a}, z_{b}\right)$ into partial subintervals of length $2 \Delta z$, where $\Delta z$ is the absolute error in the measurement of separations equal to $0.6 \mathrm{~nm}$ and $0.8 \mathrm{~nm}$ in $[15,16]$, respectively. In so doing, each subinterval $k$ contains a group of $m_{k}$ points $z_{i j} \equiv z_{k l}, 1 \leqslant l \leqslant m_{k}$ (in [15] $m_{k}$ ranges from 3 to 13). Inside each subinterval all points $z_{k l}$ can be considered as equivalent, because within the interval of width $2 \Delta z$ the value of absolute separation is distributed uniformly. The mean and the variance of the mean of the physical quantity $\Pi$ for the subinterval $k$ are defined as

$$
\bar{\Pi}_{k}=\frac{1}{m_{k}} \sum_{l=1}^{m_{k}} \Pi\left(z_{k l}\right), \quad s_{\bar{\Pi}_{k}}^{2}=\frac{1}{m_{k}\left(m_{k}-1\right)} \sum_{l=1}^{m_{k}}\left[\Pi\left(z_{k l}\right)-\bar{\Pi}_{k}\right]^{2} .
$$

If all $z_{i j}=z_{i}$, i.e. the same in different sets of measurements (as in [16]), the mean and the variance of the mean at each point $z_{i}$ are obtained more simply

$$
\bar{\Pi}_{i}=\frac{1}{n} \sum_{j=1}^{n} \Pi\left(z_{i j}\right), \quad s_{\bar{\Pi}_{i}}^{2}=\frac{1}{n(n-1)} \sum_{j=1}^{n}\left[\Pi\left(z_{i j}\right)-\bar{\Pi}_{i}\right]^{2} .
$$

Direct calculation shows that the mean values $\bar{\Pi}_{k}, \bar{\Pi}_{i}$ are uniform, i.e. change smoothly with the change of $k, i$. The variances of the mean, $s_{\bar{\Pi}_{k}}, s_{\bar{\Pi}_{i}}$, are, however, not uniform. To smooth them, we have used a special procedure developed in mathematical statistics [17, 18]. 
At each separation $z_{0}$, in order to find the uniform variance of a mean, we consider not only one subinterval containing $z_{0}$, but also several neighbouring subintervals from both sides of $z_{0}$ (4 or 5 in [15]) or about 30 neighbouring points in [16]. The number of neighbouring subintervals or points is denoted by $N$. Then the smoothed variance of the mean at a point $z_{0}$ is given by $[17,18]$

$$
s_{\bar{\Pi}}^{2}\left(z_{0}\right)=\max \left[N \sum_{k=1}^{N} \lambda_{k}^{2} s_{\bar{\Pi}_{k}}^{2}\right],
$$

where $\lambda_{k}$ are the statistical weights. The maximum in equation (3) is taken over two sets of coefficients, $\lambda_{k}=1 / N$ and $\lambda_{k}=1 /\left(c_{k} \sum_{i=1}^{N} c_{i}^{-1}\right)$, where the constants $c_{i}$ are determined from $s_{\bar{\Pi}_{1}}^{2}: s_{\bar{\Pi}_{2}}^{2}: \ldots: s_{\bar{\Pi}_{N}}^{2}=c_{1}: c_{2}: \ldots: c_{N}$. Note that max in equation (3) leads to the most conservative result, i.e. overestimates the random error. Finally, the confidence interval at a confidence probability $\beta$ takes the form

$$
\left[\bar{\Pi}\left(z_{0}\right)-\Delta^{\text {rand }} \Pi\left(z_{0}\right), \bar{\Pi}\left(z_{0}\right)+\Delta^{\text {rand }} \Pi\left(z_{0}\right)\right],
$$

where the random absolute error in the measurement of the quantity $\Pi$ at a separation $z_{0}$ is given by

$$
\Delta^{\mathrm{rand}} \Pi\left(z_{0}\right)=s_{\bar{\Pi}}\left(z_{0}\right) t_{(1+\beta) / 2}\left(\min m_{k}-1\right) .
$$

Here the value of $t_{p}(f)$ can be found in tables for Student's $t$-distribution. For example, in the experiment [16], $\min m_{k}=n=65$. Thus, for $\beta=0.95$, we have $t_{p}(f)=2.00$ and $\Delta^{\text {rand }} F^{\exp }=3.0 \mathrm{pN}$ independent of $z_{0}$.

The computational results for the relative random errors $\delta^{\text {rand }} \Pi=\Delta^{\text {rand }} \Pi /|\Pi|$ in the experiments $[15,16]$ at $95 \%$ confidence are shown in columns labelled (a) in table 1 , as functions of separation. As is seen from column 2 in table 1, in the experiment [15] the relative random error of the Casimir pressure measurements is equal to $1.5 \%$ at $z=160 \mathrm{~nm}$, then it quickly decreases to $0.4 \%$ at $z=350 \mathrm{~nm}$, and then increases with further increase of separation. This is explained by the fact that the absolute random error in equation (5) takes a maximum value at the shortest separation and monotonically decreases with the increase of separation until $z=400 \mathrm{~nm}$ [15]. At larger separations $\Delta^{\text {rand }} P^{\exp }$ is practically constant and the increase of $\delta^{\text {rand }} P^{\exp }$ is explained solely in terms of the decrease of the Casimir pressure magnitude. In the experiment [16] (column 8 in table 1), the relative random error is only $0.78 \%$ at the shortest separation $z=62.33 \mathrm{~nm}$, and quickly increases with separation due to the decrease of the Casimir force.

\subsection{Systematic errors}

In each of the experiments $[15,16]$, there are several absolute systematic errors $\Delta_{i}^{\text {syst }} \Pi(z)$ and respective relative systematic errors $\delta_{i}^{\text {syst }} \Pi(z)=\Delta_{i}^{\text {syst }} \Pi(z) /|\Pi(z)|$, where $1 \leqslant i \leqslant J$. Systematic errors are the random quantities characterized by a uniform distribution. Because of this, the total systematic error is [19]

$$
\delta^{\mathrm{syst}} \Pi(z)=\min \left\{\sum_{i=1}^{J} \delta_{i}^{\mathrm{syst}} \Pi(z), k_{\beta}^{(J)} \sqrt{\sum_{i=1}^{J}\left[\delta_{i}^{\mathrm{syst}} \Pi(z)\right]^{2}}\right\},
$$

where $\beta$ is the confidence probability and $k_{\beta}^{(J)}$ is a tabulated coefficient [19]. The same rule is also valid for the absolute systematic errors.

In the experiment [15], there are $J=2$ main systematic errors

$$
\delta_{1}^{\text {syst }} P^{\exp }=\delta R=\frac{\Delta R}{R}, \quad \delta_{2}^{\text {syst }} P^{\exp }(z)=\delta\left(\omega_{r}-\omega_{0}\right)=\frac{\Delta \omega_{r}}{\left|\omega_{r}-\omega_{0}\right|},
$$


Table 1. Relative errors $(\%)$ in experiments $[15,16]$ : random errors $\delta^{\text {rand }} P^{\exp }, \delta^{\text {rand }} F^{\exp }$ (a); systematic errors $\delta^{\text {syst }} P^{\exp }, \delta^{\text {syst }} F^{\exp }(\mathrm{b})$; total experimental errors $\delta^{\text {tot }} P^{\exp }, \delta^{\text {tot }} F^{\exp }$ (c); theoretical errors $\delta_{0} P^{\text {th }}, \delta_{0} F^{\text {th }}(\mathrm{d})$; total theoretical errors $\delta^{\text {tot }} P^{\text {th }}, \delta^{\text {tot }} F^{\text {th }}(\mathrm{e})$. Columns labelled (f) contain $\Xi_{P} /\left|\bar{P}^{\exp }\right|$ and $\Xi_{F} /\left|\bar{F}^{\exp }\right|$ (see text).

\begin{tabular}{|c|c|c|c|c|c|c|c|c|c|c|c|c|}
\hline \multirow[t]{2}{*}{$z(\mathrm{~nm})$} & \multicolumn{6}{|c|}{ Experiment of [15] } & \multicolumn{6}{|c|}{ Experiment of [16] } \\
\hline & (a) & (b) & (c) & (d) & (e) & (f) & (a) & (b) & (c) & (d) & (e) & (f) \\
\hline 62.33 & & & & & & & 0.78 & 0.31 & 0.87 & 0.55 & 3.5 & 4.0 \\
\hline 70 & & & & & & & 1.1 & 0.42 & 1.2 & 0.56 & 3.2 & 3.7 \\
\hline 80 & & & & & & & 1.6 & 0.60 & 1.7 & 0.56 & 2.8 & 3.7 \\
\hline 90 & & & & & & & 2.1 & 0.84 & 2.4 & 0.56 & 2.6 & 3.9 \\
\hline 100 & & & & & & & 2.9 & 1.1 & 3.2 & 0.56 & 2.4 & 4.4 \\
\hline 120 & & & & & & & 4.7 & 1.8 & 5.3 & 0.56 & 2.0 & 6.2 \\
\hline 140 & & & & & & & 7.3 & 2.8 & 8.1 & 0.57 & 1.8 & 9.1 \\
\hline 160 & 1.4 & 0.15 & 1.4 & 0.56 & 1.6 & 2.4 & 10 & 4.1 & 12 & 0.58 & 1.6 & 13 \\
\hline 170 & 0.59 & 0.15 & 0.59 & 0.56 & 1.6 & 1.9 & 12 & 4.9 & 14 & 0.58 & 1.6 & 15 \\
\hline 180 & 0.57 & 0.15 & 0.57 & 0.57 & 1.5 & 1.8 & 15 & 5.7 & 16 & 0.58 & 1.5 & 18 \\
\hline 200 & 0.55 & 0.16 & 0.56 & 0.57 & 1.4 & 1.7 & 20 & 7.7 & 22 & 0.59 & 1.4 & 23 \\
\hline 250 & 0.48 & 0.20 & 0.54 & 0.58 & 1.2 & 1.5 & 37 & 14 & 41 & 0.61 & 1.3 & 42 \\
\hline 300 & 0.44 & 0.31 & 0.59 & 0.59 & 1.1 & 1.4 & 62 & 24 & 69 & 0.64 & 1.2 & 70 \\
\hline 350 & 0.40 & 0.50 & 0.72 & 0.61 & 1.0 & 1.4 & 96 & 37 & 107 & 0.67 & 1.1 & 108 \\
\hline 400 & 0.56 & 0.80 & 1.1 & 0.62 & 0.98 & 1.6 & & & & & & \\
\hline 500 & 1.3 & 1.80 & 2.5 & 0.66 & 0.91 & 2.9 & & & & & & \\
\hline 600 & 2.9 & 3.80 & 5.4 & 0.70 & 0.88 & 5.4 & & & & & & \\
\hline
\end{tabular}

where $R=(148.7 \pm 0.2) \mu \mathrm{m}$ is the sphere radius, $\omega_{r}$ and $\omega_{0}$ are the resonant and natural angular frequencies of the oscillator, respectively (the former is separation dependent). $\omega_{0}=2 \pi \times 702.92 \mathrm{~Hz}$ was determined so precisely that its error does not contribute to the results, and the error of the resonant frequency is $\Delta \omega_{r}=2 \pi \times 6 \mathrm{mHz}$. Using the value $k_{0.95}^{(2)}=1.10$ and utilizing equation (6) one obtains the total systematic errors given in column 3 (labelled (b)) in table 1.

The experiment [16] contains the following $J=4$ systematic errors: $\Delta_{1}^{\text {syst }} F^{\text {exp }} \approx 0.82 \mathrm{pN}$ due to force calibration; $\Delta_{2}^{\text {syst }} F^{\exp } \approx 0.55 \mathrm{pN}$ due to noise when the calibration voltage is applied to the cantilever; $\Delta_{3}^{\text {syst }} F^{\exp } \approx 0.31 \mathrm{pN}$ due to the instrumental sensitivity; and $\Delta_{4}^{\text {syst }} F^{\exp } \approx 0.12 \mathrm{pN}$ due to the restrictions on computer resolution of data. Combining these errors using the analogue of equation (6) with $k_{0.95}^{(4)}=1.12$, we obtain $\Delta^{\text {syst }} F^{\text {exp }}=1.17 \mathrm{pN}$. The respective relative errors $\delta^{\text {syst }} F^{\exp }=\Delta^{\text {syst }} F^{\exp } /\left|F^{\exp }\right|$ are shown in column 9 in table 1 . Comparing columns labelled (b) in table 1, we conclude that in both experiments the relative systematic error increases as the separation increases. The magnitudes of the systematic errors are smaller in the experiment of [15].

\subsection{Total experimental error}

To find the total experimental error in the measurements of $\Pi(z)$, one should combine the random and systematic errors obtained above which are described by a normal (or Student) distribution and a combination of uniform distributions, respectively. To be very conservative, we assume that the systematic error is described by a uniform distribution (other assumptions lead to smaller total error). Different methods for combining random and systematic errors are described in [19]. Here we use one based on the value of the quantity $r(z)=\Delta^{\text {syst }} \Pi(z) / s_{\bar{\Pi}}(z)$. 
According to this method, at all $z$, where $r(z)<0.8$, the contribution from the systematic error is negligible and $\Delta^{\text {tot }} \Pi(z)=\Delta^{\text {rand }} \Pi(z)$ at $95 \%$ confidence. If $r(z)>8$ is valid, the random error is negligible and at $95 \%$ confidence $\Delta^{\text {tot }} \Pi(z)=\Delta^{\text {syst }} \Pi(z)$. In the separation region where $0.8 \leqslant r(z) \leqslant 8$, the combination of errors is performed using the rule

$$
\Delta^{\mathrm{tot}} \Pi(z)=q_{\beta}(r)\left[\Delta^{\mathrm{rand}} \Pi(z)+\Delta^{\mathrm{syst}} \Pi(z)\right],
$$

where the coefficient $q_{\beta}(r)$ with $\beta=0.95$ varies between 0.71 and 0.81 . Being conservative, here we use $q_{\beta}(r)=0.8$ in all calculations.

Table 1 (columns 4 and 10 labelled (c)) contains the total experimental error of the Casimir pressure and force measurements in the experiments $[15,16]$, respectively. As seen in column 4 of table 1, in the experiment [15] at $z=160 \mathrm{~nm}$ the total experimental error is equal to $1.4 \%$, but in a wide separation range from 170 to $300 \mathrm{~nm}$, it is practically flat and within the range from 0.54 to $0.59 \%$. Even at $z=600 \mathrm{~nm}$ it is equal to only $5.4 \%$. In the experiment [16] (column 10 in table 1) the smallest total experimental error of $0.87 \%$ is achieved $z=62.33 \mathrm{~nm}$ and increases up to $5.3 \%$ at $z=120 \mathrm{~nm}$. This is mainly due to the large contribution of the random errors.

\section{Determination of the theoretical errors}

The theoretical values of $\Pi(z)$ (both the pressure and force) are computed using the Lifshitz formula (see, e.g. [3]) which takes into account the effects of finite conductivity and nonzero temperature. The Lifshitz formula contains the reflection coefficients at imaginary Matsubara frequencies. At zero Matsubara frequency these coefficients are expressed in terms of the Drude dielectric function (the Drude model approach [20,21]) or in terms of the Leontovich surface impedance (the impedance approach [22,23]). At nonzero Matsubara frequencies both approaches use the tabulated optical data extrapolated to low frequencies by the imaginary part of the Drude dielectric function. In [24, 25], the reflection coefficients at all Matsubara frequencies were expressed using the free electron plasma model (the plasma model approach).

One error in the theoretical computation arises from sample to sample variations of the optical data for the complex index of refraction. Usually these data are not measured in each individual experiment, but are taken from tables. In [14], it was shown that variation of the optical data for typical samples leads to a relative theoretical error $\delta_{1} \Pi^{\text {th }}(z)$ in the computed Casimir pressure or force that is no larger than $0.5 \%$. Being conservative, we set $\delta_{1} \Pi^{\text {th }}(z)=0.5 \%$ at all separations. Strictly speaking, there may occur rare samples with up to $2 \%$ deviations in the Casimir pressure or force at short separations. If this happens, the theoretical values come into conflict with the experimental data. Such deviations must be considered not as an error (they can only diminish the magnitudes of the pressure or force) but as a correction. The validity of the hypothesis on the presence of such types of corrections can be easily verified statistically.

Another theoretical error is caused by the use of the proximity force theorem [26]. (This is the name given by the authors of [26]; some other authors, e.g. in [27], prefer to use the name 'proximity force approximation' to underline the approximate character of the equality proposed in [26].) In the experiment [15], it is applied to express the effective Casimir pressure between two parallel plates through the derivative of the force acting between a sphere and a plate. In the experiment [16], the basic result for the force is obtained using the proximity force theorem. The upper limit of error introduced by this is $\delta_{2} \Pi^{\text {th }}(z)=z / R$ [3] (see also $[27,28]$ where the same estimation was confirmed for the case of a massless scalar field).

Both errors $\delta_{i} \Pi^{\text {th }}$ are described by a uniform distribution and in this sense can be likened to systematic errors. They are combined by using equation (6) with $J=2$ leading to the values 
$\delta_{0} \Pi^{\text {th }}$ presented in columns 5 and 11 in table 1 (labelled (d)) for the experiments $[15,16]$, respectively. As is seen from these columns, the errors $\delta_{0} \Pi^{\text {th }}(z)$ depend only slightly on separation and take similar values between $0.55 \%$ and $0.70 \%$.

In addition to the major theoretical errors $\delta_{i} \Pi^{\text {th }}(z)$, there exist other uncertainties in calculations which are not taken into account in the Lifshitz formula. Some of them were shown to be negligibly small (like the contributions from patch potentials, nonlocal effects and finite sizes of the plates $[14,15])$. As to the contribution from the surface roughness, it was calculated using the atomic force microscope images of the interacting surfaces and taken into account as a correction [14-16]. This is why these factors do not contribute to the balance of theoretical errors.

There is one more error which can be considered together with the theoretical errors if one is going to compare the experimental and theoretical values of $\Pi(z)[15,16]$. This arises from the fact that $z$ is determined experimentally with an error $\Delta z$ (see section 2.1), and this error results in the additional uncertainties $\delta_{3} \Pi^{\text {th }}(z)$ in computations. Bearing in mind the leading theoretical dependences of the pressure and force on separation, we obtain $\delta_{3} P^{\text {th }}(z)=4 \Delta z / z$ in [15] and $\delta_{3} F^{\text {th }}(z)=\Delta R / R+3 \Delta z / z$ in [16]. Taking into account that the combined random quantity $\delta_{0} \Pi^{\text {th }}(z)$ may be distributed nonuniformly, we combine it with $\delta_{3} \Pi^{\text {th }}(z)$ using equation (8) and obtain the total theoretical error $\delta^{\text {tot }} \Pi^{\text {th }}(z)$ at $95 \%$ confidence. The values of $\delta^{\text {tot }} \Pi^{\text {th }}(z)$ are presented in columns 6 and 12 in table 1 (labelled (e)) for the experiments $[15,16]$, respectively. For both experiments they monotonically decrease with separation and take the largest values at the shortest separation. The significant increase of the total theoretical error in columns labelled (e) compared to those labelled (d) is due to the additional error $\delta_{3} \Pi^{\text {th }}(z)$.

\section{Comparison between experiment and theory}

\subsection{Measure of agreement between experiment and theory}

In sections 2.3 and 3, we have obtained the total experimental and theoretical errors at $95 \%$ confidence for both the Casimir pressure and force. Now we consider the new random quantity $P^{\text {th }}(z)-P^{\exp }(z)$ (or $F^{\text {th }}(z)-F^{\exp }(z)$ ) and determine the absolute error of this quantity, $\Xi_{P, F}(z)$, at $95 \%$ confidence using the composition rule (6) with $J=2$

$\Xi_{P}(z)=\min \left\{\Delta^{\text {tot }} P^{\text {th }}(z)+\Delta^{\text {tot }} P^{\exp }(z), 1.1 \sqrt{\left[\Delta^{\text {tot }} P^{\text {th }}(z)\right]^{2}+\left[\Delta^{\text {tot }} P^{\exp }(z)\right]^{2}}\right\}$

(the same equation is valid for the force). Note that in equation (9), the conservative value of $k_{0.95}^{(2)}=1.1$ is used as for two uniform distributions (otherwise it would be smaller).

The confidence interval for the quantity $P^{\text {th }}(z)-P^{\exp }(z)$ at $95 \%$ confidence is given by $\left[-\Xi_{P}(z), \Xi_{P}(z)\right]$ and the mean values $\left\langle P^{\text {th }}(z)-P^{\exp }(z)\right\rangle$ or $\left\langle F^{\text {th }}(z)-F^{\exp }(z)\right\rangle$ must belong to this interval or its analogue for the force with a $95 \%$ probability. The values of $\Xi_{P}(z) /\left|\bar{P}^{\exp }\right|$ and $\Xi_{F}(z) /\left|\bar{F}^{\text {exp }}\right|$ are given in columns 7 and 13 in table 1 (labelled (f)) for the experiments $[15,16]$, respectively. They characterize the sensitivity of the experiments $[15,16]$ to the differences between theory and experiment at 95\% confidence. For example, in [15] theory is in agreement with experiment at a separation $z=400 \mathrm{~nm}$ if $\left|P^{\text {th }}(z)-\bar{P}^{\exp }(z)\right|$ does not exceed $1.6 \%$ of $\left|\bar{P}^{\exp }(z)\right|$.

\subsection{Comparison of experiment and theory in the determination of the Casimir pressure between Au plates in [15]}

Experiment [15] is rather sensitive and can be compared with different theoretical approaches to the calculation of the Casimir pressure. The main results are presented in table 2 
Table 2. Comparison of the experiments of $[15,16]$ with theory. Columns (a) contain the absolute errors $\Xi_{P, F}(z)$ of the pressure $(\mathrm{mPa})$ and force $(\mathrm{pN})$ differences at $95 \%$ confidence. Column (b) contains the same quantity for the pressure at $99 \%$ confidence. Other columns contain the mean values $\left\langle P^{\text {th }}(z)-P^{\exp }(z)\right\rangle$ in $\mathrm{mPa}$ (and also $\left\langle F^{\text {th }}(z)-F^{\exp }(z)\right\rangle$ in $\mathrm{pN}$ for the last column labelled (e)) computed using four different approaches: the impedance (c) and the plasma model (d) approach at $T=300 \mathrm{~K}$; the optical data in the Lifshitz formula at $T=0$ (e); the Drude model approach at $T=300 \mathrm{~K}$ (f).

\begin{tabular}{|c|c|c|c|c|c|c|c|c|}
\hline \multirow[t]{2}{*}{$z(\mathrm{~nm})$} & \multicolumn{6}{|c|}{ Experiment of [15] } & \multicolumn{2}{|c|}{ Experiment of [16] } \\
\hline & (a) & (b) & (c) & (d) & (e) & (f) & (a) & (e) \\
\hline 62.33 & & & & & & & 15.2 & -0.5 \\
\hline 70 & & & & & & & 10.4 & 3.0 \\
\hline 80 & & & & & & & 7.1 & 3.6 \\
\hline 90 & & & & & & & 5.4 & 1.0 \\
\hline 100 & & & & & & & 4.5 & 2.0 \\
\hline 120 & & & & & & & 3.9 & -0.15 \\
\hline 140 & & & & & & & 3.8 & 0.02 \\
\hline 170 & 17.2 & 39.8 & 2.01 & 13.0 & 3.87 & 18.8 & 3.7 & -0.82 \\
\hline 180 & 13.4 & 31.0 & -0.74 & 7.54 & 1.24 & 14.4 & 3.7 & -0.48 \\
\hline 200 & 8.59 & 19.8 & -1.21 & 5.3 & 0.63 & 11.0 & 3.7 & -0.31 \\
\hline 250 & 3.34 & 7.72 & -0.31 & 1.3 & 0.93 & 7.09 & 3.7 & -0.84 \\
\hline 300 & 1.59 & 3.67 & 0.34 & 0.6 & 1.12 & 5.07 & 3.7 & 0.46 \\
\hline 350 & 0.89 & 2.06 & 0.38 & 0.39 & 0.80 & 3.58 & 3.7 & 0.27 \\
\hline 400 & 0.63 & 1.46 & 0.28 & 0.20 & 0.68 & 2.59 & & \\
\hline 500 & 0.49 & 1.13 & 0.11 & 0.05 & 0.32 & 1.37 & & \\
\hline 600 & 0.46 & 1.06 & 0.08 & 0.04 & 0.17 & 0.82 & & \\
\hline 700 & 0.46 & 1.06 & 0.02 & -0.01 & 0.08 & 0.51 & & \\
\hline
\end{tabular}

where the second and third columns labelled (a), (b) contain the half-width $\Xi_{P}(z)$ of the confidence interval at $95 \%$ and $99 \%$ confidence, respectively. In columns 4-7 labelled (c), (d), (e) and (f) the results for the mean differences $\left\langle P^{\text {th }}(z)-P^{\exp }(z)\right\rangle$ are computed using the impedance $[15,22]$ and the plasma model $[15,24,25]$ approach at $T=300 \mathrm{~K}$, the optical data in the Lifshitz formula at $T=0$ [3], and the Drude model approach at $T=300 \mathrm{~K}$ [20, 21], respectively. To avoid confusion, recall that in column (c) the zero-frequency contribution to the Lifshitz formula is computed using the Leontovich impedance in the region of infrared optics. At all other Matsubara frequencies the impedance is obtained using the tabulated optical data. Comparing columns 4-6 and columns 2,3, we conclude that the impedance approach, the plasma model approach and the Lifshitz formula at $T=0$ are consistent with the measurement data. At the same time, by comparing columns 2,3 with column 7 we find that the Drude model approach is excluded by experiment at $95 \%$ confidence within the separation range from 170 to $700 \mathrm{~nm}$, and at $99 \%$ confidence from 300 to $500 \mathrm{~nm}$. The physical reasons for the failure of the Drude model approach and the advantages of the Leontovich impedance are discussed in [22, 23, 29].

\subsection{Comparison of experiment and theory in measuring the Casimir force between an $A u$ sphere and a Si plate in [16]}

Experiment [16] is the first demonstration of the Casimir force between a metal and a semiconductor performed at shorter separations than in experiment [15]. For this reason it cannot be used to discriminate among different theories. In column 8 , in table 2 labelled (a) the values of $\Xi_{F}(z)$ for the force at $95 \%$ confidence are given. Column 9 in table 2 labelled 
(e) contains the values of $\left\langle F^{\text {th }}(z)-F^{\exp }(z)\right\rangle$ computed using the Lifshitz formula at $T=0$ and tabulated optical data for $\mathrm{Au}$ and $\mathrm{Si}$. The comparison of these columns shows that the theory at $T=0$ is in a very good agreement with experiment.

\section{Conclusions}

From the above, several conclusions can be reached:

- A new method for data processing and comparing theory with experiment for the Casimir effect has been presented based on rigorous results of mathematical statistics with no recourse to the previously used root-mean-square deviation.

- The distinguishing feature of this method is the independent determination of the total experimental and theoretical errors and of the confidence interval for differences between calculated and measured values at a chosen confidence probability.

- The developed method is conservative and guarantees against underestimation of errors and uncertainties. It was applied to two recent experiments measuring the Casimir pressure and force in different configurations.

- We have demonstrated that the approaches based on the vanishing contribution of the transverse electric mode at zero frequency (e.g., the Drude model approach) are excluded by experiment at $99 \%$ confidence, whereas the three traditional approaches to the thermal Casimir force are consistent with experiment.

\section{Acknowledgments}

The work of GLK, FC, UM and VMM was supported by the NSF Grant no PHY0355092 and DOE Grant no DE-FG02-04ER46131. EF was supported by DOE Grant no DE-AC0276ER071428.

\section{References}

[1] Sparnaay M J 1958 Physica 24751

[2] Van Blokland P H G and Overbeek J T G 1978 J. Chem. Soc. Faraday Trans. 742637

[3] Bordag M, Mohideen U and Mostepanenko V M 2001 Phys. Rep. 3531

[4] Lamoreaux S K 1997 Phys. Rev. Lett. 785

[5] Mohideen U and Roy A 1998 Phys. Rev. Lett. 814549

[6] Roy A and Mohideen U 1999 Phys. Rev. Lett. 824380

[7] Roy A, Lin C-Y and Mohideen U 1999 Phys. Rev. D $60111101(\mathrm{R})$

[8] Harris B W, Chen F and Mohideen U 2000 Phys. Rev. A 62052109

[9] Ederth T 2000 Phys. Rev. A 62062104

[10] Chan H B, Aksyuk V A, Kleiman R N, Bishop D J and Capasso F 2001 Science 2911941

[11] Bressi G, Carugno G, Onofrio R and Ruoso G 2002 Phys. Rev. Lett. 88041804

[12] Chen F, Mohideen U, Klimchitskaya G L and Mostepanenko V M 2002 Phys. Rev. Lett. 88101801

[13] Decca R S, Fischbach E, Klimchitskaya G L, Krause D E, López D and Mostepanenko V M 2003 Phys. Rev. D 68116003

[14] Chen F, Mohideen U, Klimchitskaya G L and Mostepanenko V M 2004 Phys. Rev. A 69022117

[15] Decca R S, López D, Fischbach E, Klimchitskaya G L, Krause D E and Mostepanenko V M 2005 Ann. Phys. NY 31837

[16] Chen F, Mohideen U, Klimchitskaya G L and Mostepanenko V M 2005 Phys. Rev. A 72 020101(R)

[17] Brownlee K A 1965 Statistical Theory and Methodology in Science and Engineering (New York: Wiley)

[18] Cochran W G 1954 Biometrics 10101

[19] Rabinovich S G 2000 Measurement Errors and Uncertainties (New York: Springer)

[20] Boström M and Sernelius B E 2000 Phys. Rev. Lett. 844757

[21] Brevik I, Aarseth J B, Høye J S and Milton K A 2005 Phys. Rev. E 71056101 
[22] Geyer B, Klimchitskaya G L and Mostepanenko V M 2003 Phys. Rev. A 67062102

[23] Bezerra V B, Klimchitskaya G L, Mostepanenko V M and Romero C 2004 Phys. Rev. A 69022119

[24] Genet C, Lambrecht A and Reynaud S 2000 Phys. Rev. A 62012110

[25] Bordag M, Geyer B, Klimchitskaya G L and Mostepanenko V M 2000 Phys. Rev. Lett. 85503

[26] Blocki J, Randrup J, Świątecki W J and Tsang C F 1977 Ann. Phys. NY 105427

[27] Scardicchio A and Jaffe R L 2005 Nucl. Phys. B 704552

[28] Gies H, Langfeld K and Moyaerts L 2003 J. High Energy Phys. JHEP06(2003)018

[29] Mostepanenko V M, Bezerra V B, Decca R S, Fischbach E, Geyer B, Klimchitskaya G L, Krause D E, López D and Romero C 2006 J. Phys. A: Math. Gen. 396589 